\title{
Teaching An Experiential Field Course Via Online Participatory Science Projects: A COVID-19 Case Study of a UC California Naturalist Course
}

\author{
Laci Gerhart ${ }^{1}$, Christopher Jadallah ${ }^{1}$, Sarah Angulo ${ }^{1}$, and Gregory Ira ${ }^{1}$ \\ ${ }^{1}$ University of California Davis
}

July 1, 2020

\begin{abstract}
Experience and training in field work is a critical component of undergraduate education in ecology, and many university courses incorporate field-based or experiential components into the curriculum in order to provide students hands-on experience. Due to the onset of the COVID-19 pandemic and the sudden shift to remote instruction in the spring of 2020, many instructors of such courses found themselves struggling to identify strategies for developing rigorous field activities that could be completed online, solo, and from a student's backyard. This case study illustrates the process by which one field-based course, a UC California Naturalist certification course offered at the University of California, Davis, transitioned to fully remote instruction. The transition relied on established, publicly available, online participatory science platforms (e.g., iNaturalist) to which the students contributed data and observations remotely. Student feedback on the course and voluntary continued engagement with the participatory science platforms indicates that the student perspective of the experience was on par with previous traditional offerings of the course. This case study also includes topics and participatory science resources for consideration by other faculty facing a similar transition from group field activities to remote, individual field-based experiences.
\end{abstract}

Teaching An Experiential Field Course Via Online Participatory Science Projects: A COVID19 Case Study of a UC California Naturalist Course

Authors: Laci M. Gerhart ${ }^{1}$, Christopher C. Jadallah ${ }^{1}$, Sarah S. Angulo ${ }^{2}$, Gregory C. Ira ${ }^{2}$

${ }^{1}$ University of California, Davis; ${ }^{2}$ UC California Naturalist Program, UC Agriculture and Natural Resources

\section{Introduction}

Fieldwork and field activities are an important component of courses in a variety of fields, including ecology and environmental science. Formally, 'fieldwork' can be defined as "any component of the curriculum that involves leaving the classroom and learning through first-hand experience" (Boyle et al 2007) including formal structured research, interpretive hikes, or any other outdoor observation, experience, or activity. In ecology and environmental science, fieldwork components in courses can benefit students through development of technical and transferable skills (Peasland et al 2019), increased confidence and motivation to learn (Boyle et al 2007), and increased favorable attitudes towards environmental protection (Fernández Manzanal et al 1999). Additionally, skills relating to natural history and naturalism have been specifically identified as lacking in undergraduate education and in formal training of ecology and environmental science professionals (Barrows et al 2016).

In the spring of 2020, when shelter-in-place guidelines were enacted in response to the COVID-19 pandemic, courses with fieldwork and field-based activities were faced with a conundrum: how to incorporate the educational and experiential value of fieldwork components into remote online instruction. This case study 
follows the transition from in-person ('traditional') to remote instruction of a UC California Naturalist (CalNat) certification course, Wild Davis, offered at the University of California, Davis (UC Davis). Since its inception in 2012, CalNat has offered certification courses through 56 partner organizations operating in 51 of California's 58 counties (Fig 1 ). CalNat courses have certified over 3,700 naturalists who have logged over 168,000 hours of volunteer service, contributing over $\$ 5$ million worth of environmental stewardship to the state of California (UCANR 2018). Field activities, such as class field trips and outdoor lessons, make up a significant component of the naturalist certification to support CalNat's mission of building a state-wide community of active and engaged environmental stewards.

Several CalNat courses were already underway when shelter-in-place recommendations were enacted in their regions and converted to online/remote instruction only for the remaining few sessions. Several others were scheduled to begin after shelter-in-place orders were enacted and, due to the difficulties in restructuring on short notice, opted to delay their start date or cancel the course. The Wild Davis CalNat course was scheduled to begin April 1, 2020; Yolo County and the UCD campus released shelter-in-place directives on March 18, 2020. Delaying the Wild Davis start date was not possible, given that the course is tied to the UCD academic calendar. Additionally, cancelling the course could have had negative ramifications for student credit hours in terms of full-time student status, financial aid, and spring graduation for seniors. Consequently, the authors of this paper, who represent the Wild Davis instructors, the Central Valley CalNat Community Education Specialist, and the CalNat Director, opted to restructure the course components to meet both the instructional expectations of the UCD campus and the core certification requirements of the CalNat program via remote instruction. This paper outlines the components of traditional instruction in this course, the process by which field-based components were transitioned to remote instruction (primarily relying on pre-existing publicly available participatory science projects), and offers guidance for faculty facing similar decisions in ecology and environmental science field courses.

This paper will use the term 'participatory science' to refer to publicly available programs and platforms through which amateur and non-professional scientists engage in scientific research through question development, study design, data collection and analysis, etc. While terms such as 'citizen science' are often used to describe these programs, the political connotations suggested by the word 'citizen' are at odds with the open and collaborative nature of these programs and have inspired careful consideration of the terminology (Eitzel et al 2017). While various terms (e.g., 'community science') reflect the variety in training, purpose, geographic scope, and number of participants that these projects employ, in none of them is U.S. citizenship necessary or relevant. Consequently, the authors of this paper follow the CalNat program's commitment (Angulo 2020) to inclusive language that honors the diversity of California Naturalists and California residents, and credits their role in public science initiatives.

Participatory science takes many forms in CalNat courses. Students are required to log observations on iNaturalist (discussed in the iNaturalist Project section) and participate in an additional instructor-chosen participatory science project (discussed in the Participatory Science Projects section). In addition, many students' capstone projects focus on or relate to participatory science (discussed in the Capstone Projects section), and under remote instruction, many students chose to complete their field activities at their sit spot (discussed in the Nature Journaling section), which under traditional instruction is reserved for solo observation activities.

\section{IRB/Campus review statement}

This case study was determined to be exempt from the UC Davis Institutional Review Board review process based on U.S. Department of Health and Human Services guidelines. It falls under the Quality Assurance/Quality Improvement activities exception as it pertains to assessing or improving a program (in the case, the course) and is not experimental in nature. Additionally, the manuscript was reviewed by representatives of the following campus offices to ensure compliance with student data and privacy guidelines: Center for Educational Effectiveness, Office of Information and Educational Technology, and the University Registrar (the campus FERPA officer). 


\section{Traditional Instruction of UC California Naturalist Courses and Wild Davis}

All CalNat courses share core curriculum, structure, and requirements, though each course differs slightly in its content focus and delivery. All courses use the California Naturalist Handbook (deNevers et al 2013) as a guiding text to the course and meet the following criteria:

- 40 hours of instruction: Students must attend all class sessions and field days (one absence is permitted with make-up work).

- Participation in iNaturalist: Log at least 1 observation in iNaturalist.

- Class Participatory Science Project: Participate in the project adopted by each class.

- Required Readings: Read the entire "The California Naturalist Handbook," as well as any relevant bioregional or topical publication as part of the UCANR 8000 online publication series, and complete assigned homework.

- Nature Journaling: Create a field notebook for nature journaling with entries from all field days and any personal observations.

- Capstone Projects. Complete, and present, a capstone project approved by the instructor. Capstone projects must involve 8 hours of service learning and fall into one of the following service categories: environmental stewardship, education, program support, climate and environmental justice, community resilience and adaptation, or participatory science.

- Evaluation: Complete a post-course evaluation survey.

- Volunteer Service: Seriously consider and hopefully commit to volunteering at least 40 hours a year in environmental stewardship, education, program support, climate and environmental justice, community resilience and adaptation, or participatory science. Record all hours of service (including capstone project hours) in the Volunteer Portal.

Within these requirements, individual courses may differ in terms of timing and structure and their bioregion or habitat of focus. Classes are generally small, ranging in size from 10-30, in order to support the focus on experiential, field-based, hands-on learning. Research on the CalNat courses and naturalists has shown that completing a CalNat course increases content knowledge on California ecosystems and environmental issues, raises student confidence in addressing environmental issues, and inspires long-term engagement with participatory science and environmental stewardship (Merenlender et al 2016)

This case study focuses on the CalNat course Wild Davis, offered to undergraduates enrolled at UCD. This course is somewhat unusual among CalNat courses in that it is focused on urban ecology in and around the town and campus of Davis and in that it is the only CalNat course offered for credit at a bachelor's degree-granting institution. The majority of CalNat courses focus on less anthropogenic landscapes (e.g., Sierra foothills, coastal redwood forests) and are offered by non-profit environmental organizations (ex: Yolo Basin Foundation, Tuleyome), research centers (e.g., Hopland Research and Extension Center), and nature outreach/education organizations (e.g., Chico Creek Nature Center). Several courses are offered through UC-affiliated organizations, such as the UC Natural Reserves and campus arboretums, and several others are offered for credit through various campuses of the California Community College System.

Structure of individual courses, in terms of length and meeting times, also varies. While each course must meet the 40 contact hours expectation, these hours can be structured as a week-long, full-time immersion course (e.g., Camp Ocean Pines), a 15-week course aligned with a university semester schedule, (e.g., American River College), a 10-week course aligned with a university quarter schedule, (e.g., UCD Wild Davis) or a multi-weekend approach (e.g., Calaveras Big Trees State Park), as fits the schedule of the organizers and the interests of the community. Since UCD is on the quarter system, Wild Davis is structured as a four-credit, 10 -week course offered during the spring quarter. Under traditional teaching scenarios, the class meets once a week for three hours during which approximately an hour is spent in lecture-style content delivery and explanation of the week's project, and the remainder of the class time is spent 'in the field' performing urban ecology data collection and experiments across campus. External activities account for the remainder of the contact hours and include field projects such as sit spots (in which students make observations of the same area throughout the quarter) and data analysis projects relating to data generated during in-class projects. 


\section{Restructuring Course Components For Remote Instruction}

\section{Contact Hours}

Previously, contact hours were met through a combination of traditional lecture-style class time, group field projects completed synchronously during scheduled class periods, and solo field projects completed asynchronously. Under remote instruction, contact hours were shifted to a combination of synchronous Zoom lectures to cover the week's reading from the California Naturalist Handbook and set up the weekly activity, and asynchronous individual efforts towards completion of the 'group' field project and solo field projects by students separately. See the Participatory Science Project section for details on how the field projects were converted to remote instruction. Zoom lectures were recorded and posted to the course Learning Management System (LMS, in this case Canvas) for student reference. Course points were awarded for attendance; however students who could not attend in the moment could make up these points. Learning objectives for each lecture were rephrased as questions and a student who missed a lecture could provide written responses to the questions in order to receive 'attendance' points for that lecture. This make-up assignment was used only once in the quarter.

\section{Nature Journaling}

Nature journaling in CalNat courses takes a variety of forms, including data collection and documentation, field notes and descriptions of activities completed, and sketches of urban flora and fauna, as well as narratives of observation activities and experiences. Previously in Wild Davis, nature journals were used by students for taking notes on lecture content, documenting their contribution to the group field projects (including data collection), and narrating and sketching their experiences at their sit spots.

Sit spots are a commonly-used instructional tool in CalNat courses, and involve making observations at the same location throughout the course. In Wild Davis, students visit their sit spot three times throughout the quarter, once around dawn, once at mid-day, and once around dusk, for 45 minutes each. Even prior to shelterin-place directives, sit spots were intended to be a solitary experience for the students, excluding friends, family, and electronic devices. Students practice focusing on each sense individually (with the exception of taste) to 'get to know' their location, and document their experience in words, sound recordings, sketches, and a limited number of photos (to reduce device use). Prior to remote instruction, students were encouraged to choose sit spots in an urban green space, garden, or park. Since the sit spot observations occur at times of day when students may not be comfortable being alone outdoors, any space in which the student felt safe was allowed (including backyards). After each observation, students turned in their nature journals for review by the instructor.

Under remote instruction, students were even more explicitly encouraged to choose a sit spot in which the student felt safe, and in which prolonged sitting was allowed. Under shelter-in-place directives, many city parks required visitors to be exercising and not 'loitering' which limited the options for students in 2020. Consequently, several students selected sit spots in their backyard $(n=9 / 23)$, though many still chose a neighborhood park or greenspace $(n=14 / 23)$. Unlike previous quarters, students were also allowed to change the location of their sit spot if the student's access to or comfort with the location changed. Students completed their observations in their nature journals and then 'turned in' their journal entries via scans/photographs or typed transcripts of the journal pages uploaded to the course LMS.

Under traditional instruction, sit spots are reserved for solo nature journaling and observation activities; however, under remote instruction, students often used their sit spot to complete other field activities (described in the Participatory Science Projects section). This provided an avenue for place-based learning, or learning about "local natural, built, and social environments through inquiry, environmental action, and other hands-on activities in a specific place" (Kudryavstev et al 2012, p. 240). In addition to fostering proenvironmental behavior, place-based education can foster academic achievement, positive social-emotional outcomes, and greater appreciation of the natural world (Sobel, 2005). Participation in direct instruction through synchronous lectures, in combination with engagement in multiple forms of experiential activity such as nature journaling, observation activities, and completion of field activities, represents a rigorous and 
effective strategy to strengthen students' sense of place (Kudryavstev et al 2012).

iNaturalist Project

iNaturalist is a participatory science portal in which members of the public upload photos or audio recordings of wildlife. Observations are tagged with location and time/date stamps and publicly searchable on the organization's website (inaturalist.org). Identification of organisms is assisted with an artificial intelligence interface which suggests possible taxonomic groups, as well as a robust community of users who suggest identifications and/or confirm other user's suggestions. Photos or audio recordings can be taken and uploaded in the moment on a mobile device, or with a traditional camera and uploaded later from a laptop or desktop.

All CalNat courses incorporate iNaturalist observations into at least one activity (in addition to a class participatory science project, discussed in the Participatory Science Projects section). Wild Davis students participate in the iNaturalist-focused City Nature Challenge (CNC, citynaturechallenge.org) which promotes iNaturalist observations of urban wildlife in cities around the world during a four-day weekend, in this case April 24th-27th, 2020. The $2020 \mathrm{CNC}$ was heavily impacted by shelter-in-place guidelines and limited access to regional greenspaces. Numerous participating cities opted to cancel their CNC entirely. Other cities cancelled all formal gatherings and events, and transitioned to a "City Nature Celebration," reducing the competitive focus of the CNC and emphasizing observing backyard wildlife.

Figure 2 compares CNC participation between 2019 and 2020 in both numbers of observations (Fig 2A ) and number of participants (Fig 2B ). All 2019 Wild Davis students made observations in the Sacramento Region (which includes the UCD campus and town of Davis). The majority of 2020 Wild Davis students $(21 / 23)$ resided in a CNC-participating region, and students outside these regions could still post observations to iNaturalist during the $\mathrm{CNC}$ window, though their observations were not included in CNC totals. In both quarters, students received course points for participation in the CNC (or participation on iNaturalist during the CNC for students outside CNC regions), with full points received for at least 20 observations. In 2019, students were required to make observations of wild organisms (not captive or cultivated); however, to accommodate shelter-in-place directives in 2020, cultivated plants (including landscaping and gardens) were allowed, so long as the observation was marked "Captive/Cultivated" on iNaturalist.

Likely due to limitations to outdoor recreation under shelter-in-place guidelines, overall Wild Davis participation in the CNC was reduced in 2020 compared to 2019 (Fig 2A ). Prior to the spring 2020 quarter, only one 2020 Wild Davis student had an iNaturalist account and no 2020 students participated in the 2019 CNC (Fig 2B ). Interestingly, the majority of 2020 CNC observations by Wild Davis students were made by students from the 2019 cohort $(862 / 1389,62 \%$,Fig 2A ), though this large contribution was generated by a small number $(\mathrm{n}=2)$ of individual students (Fig $\mathbf{2 B})$.

\section{Participatory Science Projects}

In Wild Davis, each week of coursework includes content from one chapter of the California Naturalist handbook and a field activity aligning with that week's content. Even in traditional instruction, a few of these activities involved participatory science programs other than iNaturalist. Under remote instruction, online participatory science projects made up half of the field activities (4/8). The remaining activities included online research projects $(2 / 8)$ and ecological observation activities not linked to an established participatory science project $(2 / 8)$.

\section{Choosing a participatory science project}

The participatory science projects used in Wild Davis in 2019 and 2020 are listed in Table $\mathbf{1}$, along with some important traits for consideration when choosing a project. The most important traits were that the students could complete the activity from an urban location (especially a backyard) without special sampling equipment or training and with limited guidance from the instructor. The lack of in-the-moment, one-on-one guidance led the instructor to select projects that assisted users with identifying the organisms or structures in their observations. Additionally, the constraint of using backyard or neighborhood locations led the instructor to select projects whose website allowed for viewing and visualizing previously-reported 
observations. In this way, even if a student's backyard was not conducive to making new observations in the project, the student could still participate in the activity by summarizing other user's observations from their region or comparing nearby regions. This secondary option for participating in the field activities was only necessary for the Debris Tracker activity, in which not all students were able to find litter or debris in their neighborhood.

Two participatory science projects were used in traditional instruction in Wild Davis in 2019 but were not used in 2020: CALeDNA, and School of Ants. CALeDNA involves collecting soil or sediment samples to mail into the project for environmental DNA (eDNA) analysis. This project has a particular sampling protocol and requires some sampling and sample storage supplies. The timeline between the shift to remote instruction and the schedule for this activity meant there was not enough time to mail individual students the supplies and so this activity was replaced. School of Ants involves setting outdoor baits and collecting the ants that are attracted to the baits to mail into the project for identification. While the sampling protocol is simple and could easily be completed in a backyard, and the supplies needed are minimal, the project does not include identification of ant species during collection (only after the samples are mailed in). When completed in traditional instruction, the instructor invites an ant expert guest speaker who can identify the ant species and share some interesting ecological, biological, or life history facts with the students. While this activity is frequently described as the students' favorite on course evaluations, the instructors did not believe it would translate well to remote instruction in this particular case and chose to replace it.

\section{Developing activities around participatory science projects}

An important component of developing course activities around participatory science projects is linking the participatory activity with course learning objectives. The content in Wild Davis lends itself rather easily to participatory science projects, making these links easier than they might be in other courses. Each week's content is structured around a single chapter of the California Naturalist Handbook for which several participatory science projects could be relevant. For example, one chapter of the Handbook broadly covers California's animal communities, including trophic interactions, functional groups, introduced and invasive animals, as well as highlights of interesting or iconic California wildlife in a variety of taxonomic groups. Participatory science projects that could be integrated into this content include (but are not limited to):

- iNaturalist: through either general wildlife observations or with respect to specific projects tracking individual taxa or regions, including invasive species.

- Taxon specific observation projects: eBird, Bumble Bee Watch, Bat Detective (through SciStarter), etc.

- Season specific projects: Nature's Notebook (through the National Phenology Network), NestWatch, etc. Courses operating in the fall could also take advantage of seasonal projects such as the Christmas Day Bird Count (through the Audubon Society)

- Region specific projects: UCD Tricolor Blackbird Project, Western Monarch Milkweed Mapper, etc

- Abiotic projects with a conceptual link to wildlife issues: GLOBE at Night (light pollution), Debris Tracker (litter and garbage), Stream Selfie (through SciStarter; water quality and flow patterns), etc. Note that projects of this nature would require more framing and perhaps additional 'research' outside of the participatory science program in order to strengthen links to course content.

Weekly points were assigned for completing each activity, which required the students to provide 'evidence' of their participation in the project. In most cases, this took the form of uploading a screenshot of their submission to the participatory science project and a short description of how and where they collected their data to the course LMS or Gradescope assignment.

In the majority of cases, the instructor contributed data or observations to the project prior to the course session in which the activity was introduced and could then share their data/observations as an example. Interacting with the data submission portal prior to assigning the activity was also important for troubleshooting problems the students might encounter in completing the activity. For example, GLOBE Cloud Observer requires submitted observations to be 'reviewed' before they are visible in the public data record. 
This extra step meant that students needed to either 1) complete the activity at least a day before the assignment deadline to ensure their observations were visible to the instructor, or 2) take a screenshot of their observation prior to submitting it to the portal, which they could share with the instructor to document their activity.

\section{Protecting student privacy in online projects}

User privacy is also an important consideration when requiring students to participate in online activities. The majority of the participatory science projects used in this class (Table $\mathbf{1}$ ) allow for anonymous reporting. Even when the project posts maps or visualizations of observations, the user who submitted the data is not always reported (e.g., GLOBE Cloud Observer). Even projects that report the observer's name allow for users to use a screen name that need not be their legal name and report no other identifying information (e.g., Debris Tracker and iNaturalist). The most robust privacy measures, illustrated by iNaturalist, allow users to obscure the geolocation of their observation, which is particularly important when students are primarily making observations in or near their home. Additionally, the option of summarizing or analyzing other user's data could be used as an option for students uncomfortable with the required online presence of participatory science projects. Indeed, research has shown that engaging with the broader dataset in which their individual observations are 'nested' has a positive impact on student valuation of participating in such projects and on students' perception of themselves as agents of environmental change (Harris et al 2020).

\section{Other activities that replaced in-person field activities}

Four of the eight field projects did not incorporate online participatory science projects. Two of these activities were short online research projects. The activity on California soils and geography asked the students to research a Superfund site (https://www.epa.gov/superfund) in their hometown or place of residence. The activity on land management asked students to research a California land trust active in their hometown or place of residence (e.g., California Council of Land Trusts; https://www.calandtrusts.org/).

Two other activities involved outdoor field observations not linked to a participatory science project. One of these was the first day's introductory activity of an urban ecology scavenger hunt in which students 'collected' ecological interactions in their backyard or neighborhood (e.g., an interaction between an animal and a plant; a mutualistic interaction, etc.). Many students completed this activity without leaving their yard; indeed, the smaller spatial scale inspired additional creativity for some of the more uncommon scavenger hunt 'items.' The second of these activities was an audio identification activity for local birds. This activity was developed specifically because several students had commented in their first sit spot observation that they heard birds they could not identify. The instructor brought in a guest speaker who has experience in teaching 'birding by ear' to help students practice describing bird calls and identifying common birds solely by their sounds. The activity for the week then focused on audio recordings from the student's sit spot in which they identified bird calls, other biotic sounds (e.g., squirrels), and anthropogenic sounds (e.g., cars, construction).

\section{Capstone Projects}

CalNat capstone projects require eight hours of outside of class service learning in support of an environmental or nature education organization. Capstone projects must fall into one of six categories: environmental stewardship or habitat restoration, education or interpretation, program support, climate and environmental justice, community resilience and adaptation, or participatory science. Under traditional in-person instruction, capstone projects have been a mix of data collection for management purposes (e.g., water quality measurements in the UC Davis Arboretum and Public Garden waterway), infographics and public signage (e.g., infographic on data collected by the Lake Tahoe Environmental Research Center's participatory science project Snapshot Day), and hands-on product development (e.g., bee nesting post for the Davis Central Park Pollinator Garden).

Under remote instruction, capstone projects needed to be coordinated and completed solo and exclusively online. These restrictions meant that the majority of capstone projects were interpretive resources such as infographics and guides (e.g., raptor ID guide for the UCD Raptor Center, https://crc.vetmed.ucdavis.edu/) 
or educational activity books for elementary age students (e.g., Take Care Tahoe activity booklet; https://takecaretahoe.org/) which could be disseminated online. Additionally, several students used the participatory science project GLOBE at Night to track patterns in urban light pollution. This project was not formally used as a group activity in the course, but worked well for capstone projects given its focus on urban observations and so is included in Table 1 .

Two capstone projects related directly to the COVID pandemic. One of these involved a "Truth About Bats" infographic developed in conjunction with the Yolo Basin Foundation (http://yolobasin.org/) to counter misinformation on the relationship between bats and disease. A second capstone project focused on mapping the location of $\mathrm{CNC}$ observations in urban centers to compare the frequency of observations in urban greenspaces versus residential locations in the 2019 CNC versus the 2020 CNC. This project was developed in conjunction with the UC Davis Center for Community and Citizen Science (https://education.ucdavis.edu/centercommunity-and-citizen-science), an organizer for the Sacramento Region CNC.

\section{Building a Sense of Community}

Since Wild Davis began after shelter-in-place directives, the course was fully online and the students and instructors never met in person. One of the most difficult aspects of fully online instruction is building a sense of community among the students and between students and the instructor. The social dimensions of learning are often underemphasized in teaching and learning, yet educational research consistently shows how social relationships are an important mediator of educational processes and outcomes (Vygotsky, 1978). Two major components of the course supported students and instructors getting to know one another: synchronous lectures, and student presentations.

The content lecture portion of the course was held synchronously on Zoom during the regularly scheduled class time. Each lecture was recorded and posted to the learning management system. All students and instructors resided in the same time zone, reducing scheduling issues. Attendance was 'required.' though students were allowed a make-up assignment (used only once in the quarter) if they were unable to attend a lecture. The consistently high level of attendance contributed to the sense of community among the instructors and students.

Students were also required to give two presentations on Zoom to the rest of the class. The first of these presentations occurred during the quarter and involved sharing the 'results' of their data collection from the week's activity. Students were allowed to choose which week they presented and students presenting the same week could either share results and present as a group, or present on their own. Participatory science projects for which submitted data were visible to users allowed students to also share the class or regional data in aggregate and compare their particular observations to others from the class or region. The second presentation was sharing the final product of their capstone project with the class and was done instead of a final exam. Since the capstone projects are completed mostly on the students' own outside of class time, this presentation served to illustrate to the group the type of capstone project each student completed, and give each student a chance to share the results of their hard work with the group. To present, students needed to unmute and speak to the group, but did not need to turn on their webcams. Students whose internet bandwidth was not reliably able to screenshare sent their presentation to the instructor who shared it for them.

\section{Comparing Student Engagement to Previous Course Offerings}

Anonymous end-of-course student evaluations indicate the course was on par with previous offerings in terms of student engagement (Table 2, Fig 4 ). While bias in student evaluations is well-documented (e.g., Stark and Freishtat 2014), many of the triggers of bias (such as those relating to instructor identity) were the same between the 2019 and 2020 cohorts; the most influential exceptions being response percentage (which was lower in 2020), and the slight change in wording of UCD campus evaluation questions.

Generally, student perspective on the course was highly positive and a transition to remote instruction resulted in minimal change in student overall ratings (Table 2 ). When asked on the evaluations to rank individual 
components of the course as most to least helpful, the 2020 cohort identified field trips and interacting with others in the class as the most beneficial components (Fig 4 ). Interestingly, these two components were ranked higher in the online 2020 course than the traditional in-person 2019 course. The survey results are consistent with anecdotal evidence from student-instructor conversations. Most commonly in these conversations, students appreciated synchronous lectures, as many of their classes were fully asynchronous and provided little opportunity for interaction. Additionally, students appreciated the opportunity, indeed the requirement, to leave their computer screen and go outside even only to their backyard for a short while. As previously mentioned, social relationships (Vygotsky, 1978) and a sense of place (Kudryavstev et al 2012) are important components of the educational environment, perhaps even more so during COVID when students were quarantined indoors and isolated from the majority of their interpersonal interactions. These results promisingly indicate that it is possible to develop engaging field components and interpersonal interaction under remote instruction and shelter-in-place directives.

The most diverging evaluation statistic between the two course offerings was the pre/post question on selfconfidence (Table 2 ). The 2019 cohort entered with very low confidence and ended near the state average for participants in other CalNat courses, while the 2020 cohort entered with higher confidence and ended extremely high. It is difficult to interpret these numbers. The unusually high 2020 pre-course values may be a result of students self-selecting, if the course attracted students who were already aware of and educated in California environmental issues, though why this would be the case in 2020 and not 2019 is unclear. The unusually high 2020 post-course values, and the unusually high gain in self-confidence from pre- to post-course, may also be related to the higher emphasis on interacting with other user data in participatory science projects. As mentioned previously, interacting with the broader dataset in which their observations are 'nested' has been shown to have a positive impact on students' perception of themselves as agents of environmental change (Harris et al 2020). Additionally, the 2020 values may have been disproportionately impacted by the low response rate to the CalNat survey and not reflective of the student body as a whole.

Another metric of student engagement relates to continued activity in the participatory science projects introduced in the course. iNaturalist provides the clearest picture of this metric, given its broad focus in terms of taxonomy and geography, its robust data access portal, and the fact that it is the most user friendly participatory science project in the course, and the one with which the students interact the most.Figure 3 shows the number of observations students in the 2019 and 2020 Wild Davis cohorts contributed to iNaturalist in the six weeks following the end of the CNC (when required activity on iNaturalist as part of the course was complete). In both years, students remained active in iNaturalist following the completion of the CNC. In both years, the majority of observations were contributed by a single 'superuser' student in the 2019 cohort whose interest in iNaturalist was unusually high. Interestingly, while activity on iNaturalist by 2020 Wild Davis students following the 2020 CNC was much lower than that of the 2019 cohort following the 2019 $\mathrm{CNC}$, it was still non-zero and maintained for the full six-week window despite continued shelter-in-place directives. These results suggest that for some students, engagement with and enjoyment of participatory science continued beyond the class project and became a part of their regular recreational activities. These results are also consistent with a resurgence in interest in community and backyard gardening during COVID (while no formal data yet exist to our knowledge, see Walljasper and Polansek 2020).

In this section, we compared student engagement in and evaluation of the fully remote 2020 offering and the traditional in-person 2019 offering of the course. We recognize that, had the students experienced both versions of the course, the remote 2020 offering may not have rated so highly. It is worth noting, however, that the majority of students experience a course only once, and so, cannot compare it to previous or subsequent offerings (as instructors can). These results indicate that students respond positively to a remotely-instructed field course that is as engaging as possible, even if it may still be less engaging than the course could be in person.

\section{Conclusion}

This case study illustrates how a field course focused on in-person group projects relating to urban ecology can be transitioned to fully remote instruction, primarily using pre-existing online participatory science 
projects. While this strategy may not serve all field courses in terms of structure or content, the diversity of readily accessible participatory science projects is robust and continuing to increase. Additionally, the limited available evidence suggests that the remote offering of the course was similar to the traditional in-person offering in terms of general engagement and student appreciation. Indeed, students valued interpersonal interaction and outdoor field components more highly in the remote instructed quarter than under traditional in-person instruction.

Faculty wishing to replace in-person field activities with pre-existing, online participatory science projects may wish to consider the following when selecting and integrating a project. The questions listed below are drawn from conversations and experiences the instructional team had as they developed and enacted activities under remote instruction. While the relevance of each consideration may vary by course content, structure, and logistics, the instructional team found that considering these points greatly aided in selecting and integrating participatory science projects into the course.

In terms of the logistics of the participatory science project:

- What experience, if any, do the students already have with the project? Students who have experience with the project will likely need less instructor guidance and support and may be able to serve as mentors or group project leaders for students with less experience.

- Does the project require particular sampling equipment? If so, this project may be difficult for students to complete if they do not have access to the equipment. The instructor may be able to mail students equipment, depending on the timeline and size of the class.

- Does the project have a complex sampling protocol? If so, it may be difficult for the students to follow the protocol accurately without one-on-one, real-time guidance.

- Does the project support data quality via guidance or identification of organisms, structures, or processes being observed? If so, the students may find it easier to gather reliable data without one-on-one, real-time guidance.

- Does the project require a mobile app for data submission? If so, students without a data plan may have difficulty participating. Can a computer or paper submission be used instead? If so, this makes the project more accessible to a wider cohort of students.

- Does the project provide user-friendly access to reported data? If so, students may be able to complete a data summary activity instead of, or in addition to, data submission. Additionally, the instructor may be able to more easily track student participation in the project.

- Does the project provide an opportunity for interaction among participants? If so, this may support the building of a sense of community in the course. For example, iNaturalist allows observers to comment on one another's observations, and to create projects for individual taxa, regions, or observers. A course-focused project allows students to easily see and comment on their colleagues' observations.

- What is the expected duration of active participation in the project? Many projects allow for singleobservation activity (e.g., iNaturalist); however, prolonged or repeated observations may be expected or required (e.g., NestWatch). Either of these may be preferred, depending on how the project integrates into the coursework.

In terms of linking with course content and assignments:

- How does participating in the activity contribute to course learning goals and course content? What does the instructor expect the students to gain by participating in the project? Example goals include 1) learning to identify certain organisms or structures (e.g., clouds), 2) practicing scientific skills such as using sampling equipment or following a protocol, 3) developing data skills such as assessing data quality or repeatability, or visualizing data, 4) understanding the process of science, such as generating and testing a hypothesis using data from the project, etc.

- How will students engage with the project and the data it generates? Options include 1) submitting individual data/observations, 2) summarizing, analyzing, or visualizing other user's data from the project (either alone or in groups), 3) comparing data from similar projects (e.g., particular species observations in iNaturalist versus eBird), etc 
- How will grades be assigned for the activity? How much participation in the project is expected for full credit? How will the instructor follow student activity or submissions in the project?

- What difficulties might the students experience in completing the activity? Are there situations in which a student may not be able to participate in the project? For this question, it is important for the instructor to test-run making observations or contributing data to the project prior to assigning the activity to students.

- How does temporal and spatial variation in observations affect the outcomes of the activity? In some cases, such variation limits the ability to 'use' the data in a meaningful way (e.g., due to low replication). In other cases, however, this variation can be useful. For example, under traditional instruction, making observations in GLOBE Cloud Observer together on campus would not make sense as all students would be observing the same clouds; however, spreading this activity across the geography of the students' residences and over a week-long timespan allowed for greater variety in cloud types observed.

There are many additional resources to assist faculty in strategizing incorporating participatory science projects into a remotely instructed course, such as the Citizen Science Association's (https://www.citizenscience.org/) series of webinars on participatory science and higher education, all of which are available on the CSA YouTube Channel (https://www.youtube.com/channel/UChTgtIf9BqiEpWiczvH0jbA). Additionally, several 'warehouse' sites exist for accessing a variety of participatory science projects, which illustrate the diversity of projects available in terms of content focus and structure of participation. These include sites such as Zooniverse (https://www.zooniverse.org/), Sci Starter (https://scistarter.org/), and CitSci.org (https://citsci.org/). SciStarter also recently released a field guide to participatory science projects geared towards a variety of age levels and content fields (Cavalier et al 2020).

\section{Literature Cited}

Angulo S. 2020. A Small Change for a Big Impact. UC California Naturalist . Published 06/23/2020, Accessed 6/29/20; Retrieved from http://calnat.ucanr.edu/blog/?blogpost $=42824 \&$ blogasset $=110130$

Barrows CW., Murphy-Mariscal M., Hernandez R.R. 2016 At a Crossroads: The nature of natural history in the twenty-first century.Professional Biologist , 66(7): 592-599.

Boyle A., Maguire S., Martin A., Milson C., Nash R., Rawlinson S., Turner A., Wurthmann S., Conchie S. 2007. Fieldwork is Good: the student perception and the affective domain. Journal of Geography in Higher Education . 31:2, p 299-317 DOI: 10.1080/03098260601063628

Cavalier D., Hoffman C., Cooper C. 2020. The Field Guide to Citizen Science: How you can contribute to scientific research and make a difference, Portland, OR, USA, Timber Press, 185

de Nevers G. Edelman D.S., Merenlender A. 2013. The California Naturalist Handbook, Berkeley, CA, USA; University of California Press

Eitzel M.V., Cappadonna J.L., Santos-Lang C., Duerr R.E., Virapongse A., West S.E., Kyba C.C.M., Bowser A., Cooper C.B., Sforzi A., Nova Metcalf A., Harris E.S., Thiel M., Haklay M., Ponciano L., Roche J., Ceccaroni L., Shilling F.M., Dorler D., Heigle F., Kiessling T., Davis B.Y., Jiang Q. 2017. Citizen Science Terminology Matters: Exploring key terms.Citizen Science: Theory and Practice . 2(1):1 pp. 1-20

Kudryavtsev, A., Stedman, R. C., \& Krasny, M. E. (2012). Sense of place in environmental education. Environmental Education Research ,18 (2), 229-250. https://doi.org/10.1080/13504622.2011.609615

Merenlender A.M., Crall A.W., Drill S., Prysby M., Ballard H. 2016 Evaluating environmental education, citizen science, and stewardship through naturalist programs. Conservation Biology . 30(6):1255-1265

Sobel, D. 2005. Place-based education: Connecting classrooms \& communities . Great Barrington, MA, USA. The Orion Society. $109 \mathrm{p}$ 
Stark P.B., Freishtat R. 2014. An evaluation of course evaluations.ScienceOpen Research DOI: 10.14293/S2199-1006.1.SOR-EDU.AOFRQA.v1

University of California, Agriculture and Natural Resources (UCANR). 2018. UC California Naturalists Program Annual Report FY16/17. Accessed 6/29/20. Retrieved from http://calnat.ucanr.edu/files/277923.pdf

Vygotsky, L. S. 1978. Mind in society: The development of higher psychological processes . Cambridge, MA, USA. Harvard University Press. 159 p

Walljasper C., Polansek T. 2020. Home gardening blooms around the world during coronavirus lockdown. Reuters . Published 04/19/2020. Accessed 6/29/20. Retrieved from https://www.reuters.com/article/ushealth-coronavirus-gardens/home-gardening-blooms-around-the-world-during-coronavirus-lockdownsidUSKBN2220D3

Tables

Table 2: Course survey and evaluation results from traditional (2019) and remote (2020) instruction

\begin{tabular}{|c|c|c|}
\hline Category or Question & Spring 2019 & Spring 2020 \\
\hline $\begin{array}{l}\text { Overall rating of instructor* } \\
\text { (via UCD course evaluations) }\end{array}$ & $4.8 \pm 0.6(\max 5)$ & $4.9 \pm 0.3(\max 5)$ \\
\hline $\begin{array}{l}\text { Overall rating of course** (via } \\
\text { UCD course evaluations) }\end{array}$ & $4.7 \pm 0.8(\max 5)$ & $4.9 \pm 0.3(\max 5)$ \\
\hline $\begin{array}{l}\text { Overall Satisfaction- (via } \\
\text { CalNat course survey, } 2018 \text { state } \\
\text { average } 92 \% \text { ) }\end{array}$ & $96 \%$ & $92 \%$ \\
\hline $\begin{array}{l}\text { Instructor Performance- (via } \\
\text { CalNat course survey, } 2018 \text { state } \\
\text { average: } 91 \% \text { ) }\end{array}$ & $100 \%$ & $100 \%$ \\
\hline $\begin{array}{l}\text { Plan to Volunteer? (via CalNat } \\
\text { course survey) }\end{array}$ & $80 \%$ & $92 \%$ \\
\hline $\begin{array}{l}\text { Self Confidence, pre/post (via } \\
\text { CalNat course survey, } 2018 \text { state } \\
\text { average: } 26 / 47 \% \text { ) }\end{array}$ & $17 / 50 \%$ & $62 / 100 \%$ \\
\hline Response rate, UCD/CalNat & $94 / 79 \%$ & $73 / 54 \%$ \\
\hline
\end{tabular}

*In the spring 2019 evaluation, this question was worded "What is your overall evaluation of the instructor: excellent (5) | above average (4) | average (3) | below average (2) | poor (1)" while in the Spring 2020 evaluation, this question was worded "Please indicate the overall teaching effectiveness of the instructor: excellent (5) | very good (4) | satisfactory (3) | fair (2) | poor (1)"

** In the spring 2019 evaluation, this question was worded "What is your overall evaluation of the course: excellent (5) | above average (4) | average (3) | below average (2) | poor (1)" while in the Spring 2020 evaluation, this question was worded "Please indicate the overall educational value of the course: excellent (5) | very good (4) | satisfactory (3) | fair (2) | poor (1)"

- Percent of respondents that responded 'satisfied' or 'very satisfied' to the prompt "Overall, how satisfied were you with the California Naturalist course you attended? Very satisfied | Satisfied | Somewhat Satisfied | Somewhat Unsatisfied | Not Satisfied"

- Percent of respondents that responded 'very good' or 'excellent' to the prompt "How would you rate the performance of your lead instructor? Excellent | Very Good | Good | Needs Improvement | Poor"

? Percent of respondents that responded 'Yes' to the prompt "As a California Naturalist, do you plan to volunteer in the coming year? Yes|No" 
Retrospective pre/post course; percent of respondents that 'strongly agree' they are "capable of making a positive impact on the environment"

\section{Data Accessibility Statement}

To ensure FERPA (Family Educational Rights and Privacy Act) compliance, the authors will share only aggregated, deidentified data upon request.

\section{Acknowledgements}

The authors would like to thank the representatives of campus offices who reviewed the manuscript for compliance with data and privacy protocols. Additionally, the authors thank the Wild Davis students in both the 2019 and 2020 cohorts for their contributions to this case study.

\section{Hosted file}

Gerhart_Table_1.docx available at https://authorea.com/users/331393/articles/465216-teachingan-experiential-field-course-via-online-participatory-science-projects-a-covid-19-casestudy-of-a-uc-california-naturalist-course 


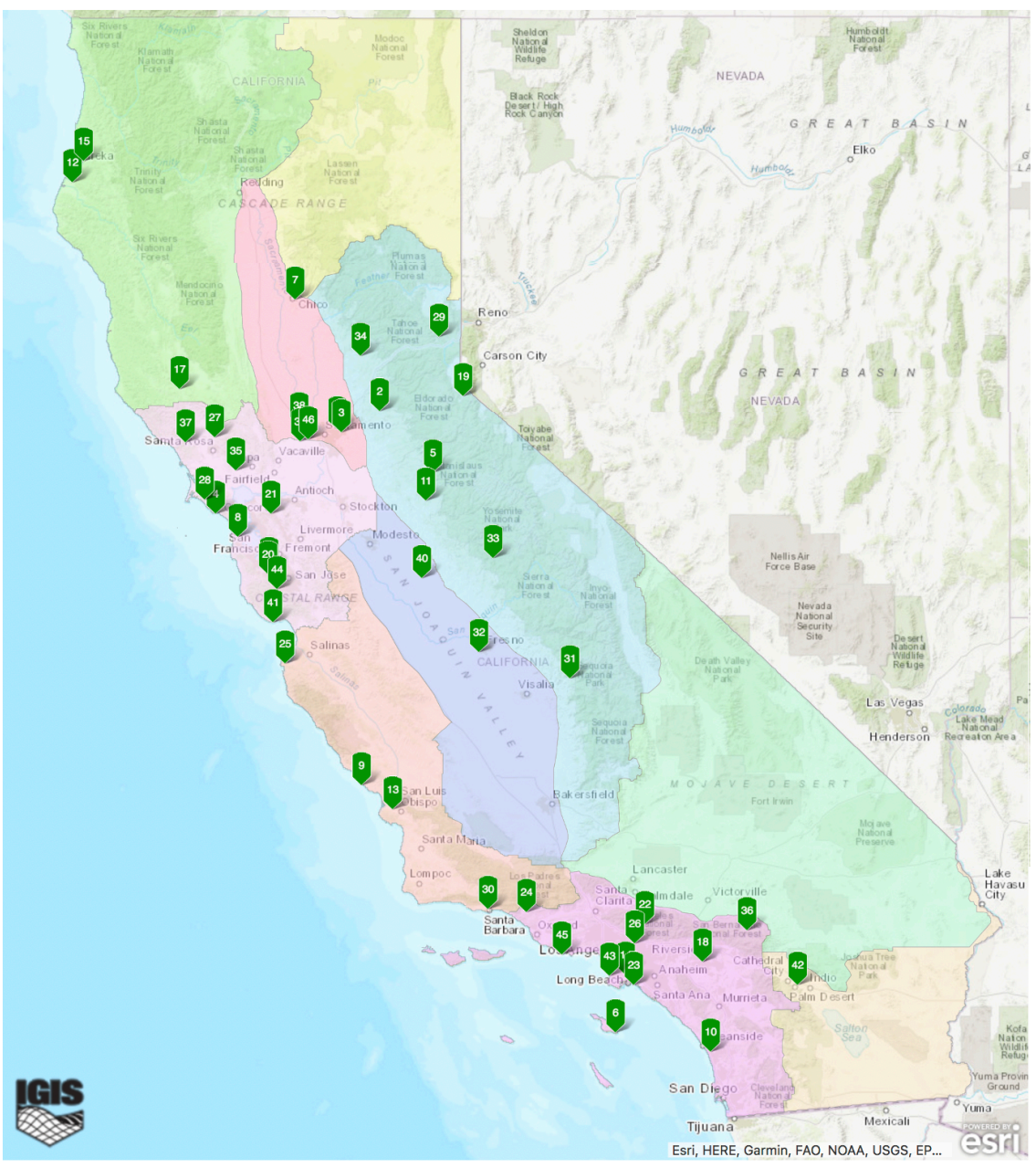




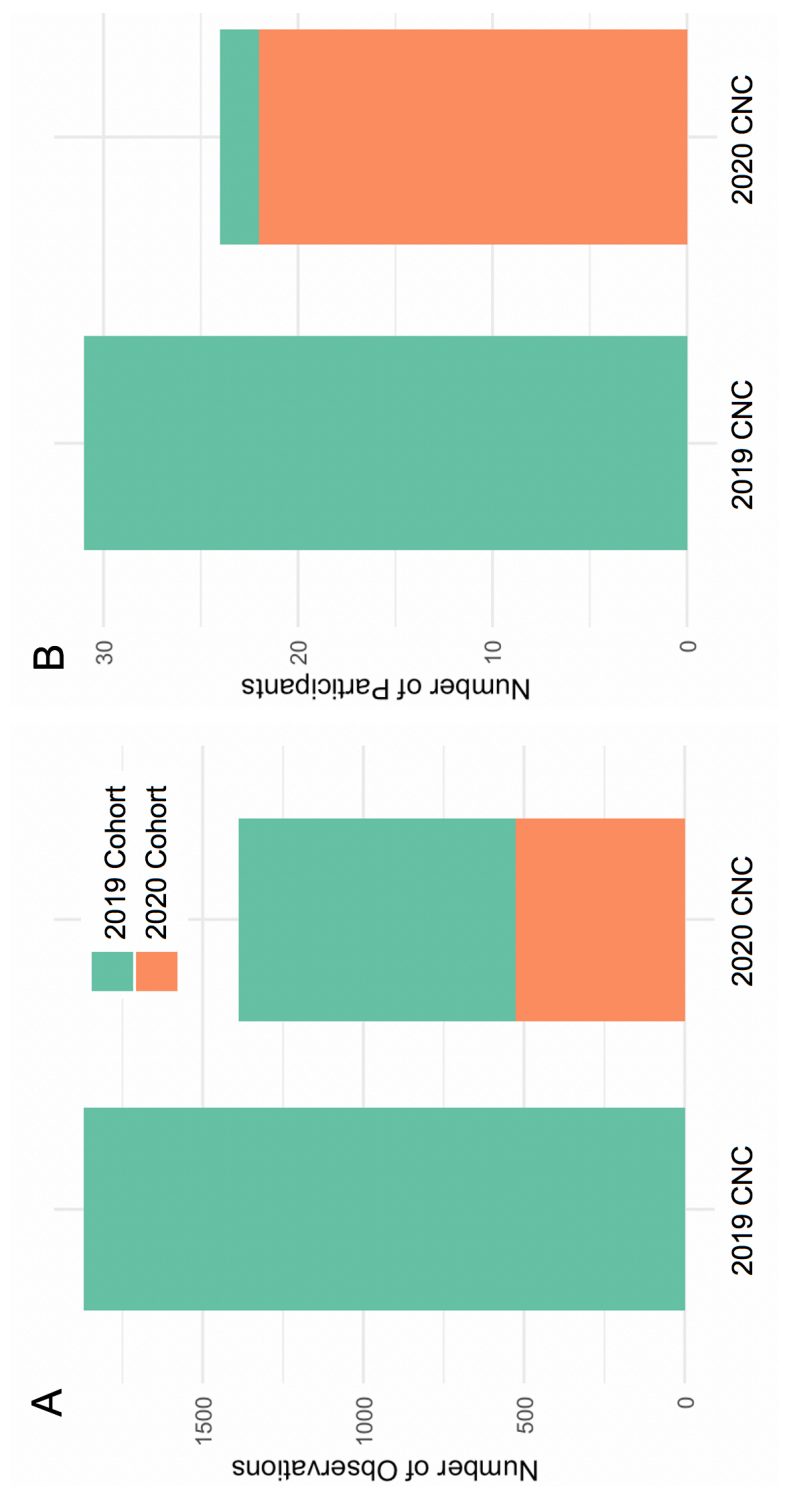




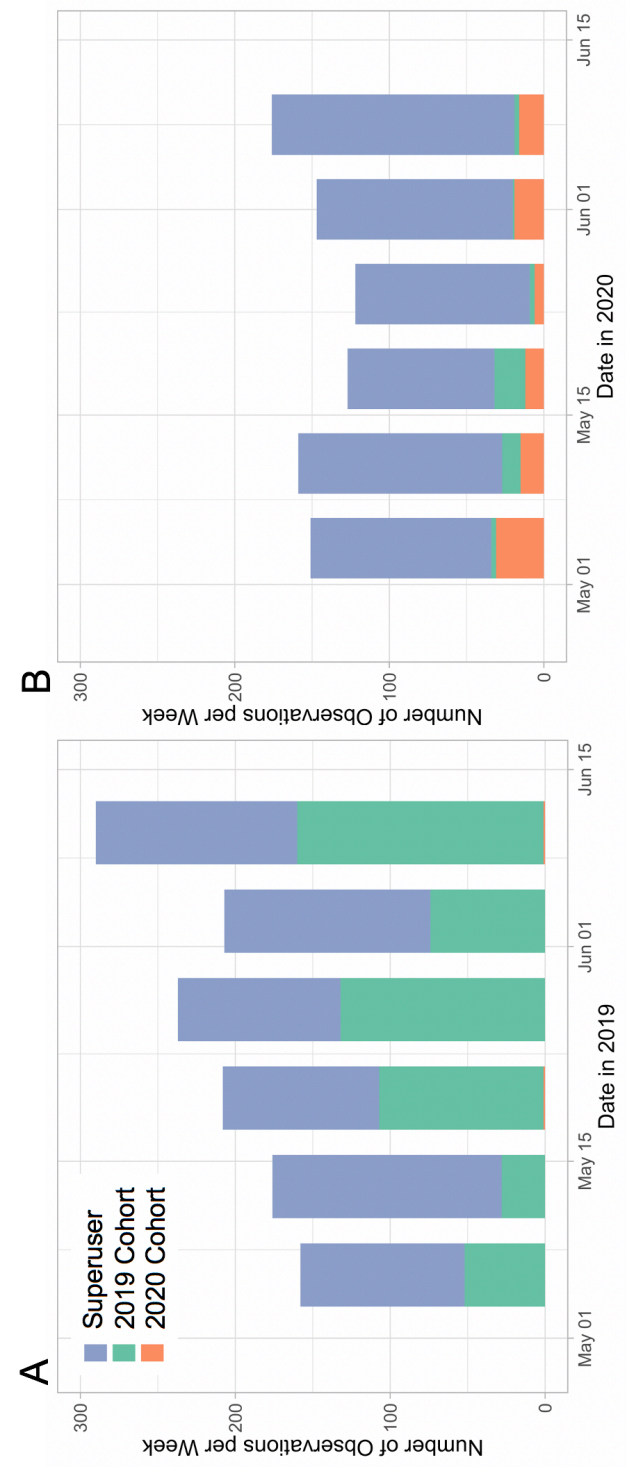




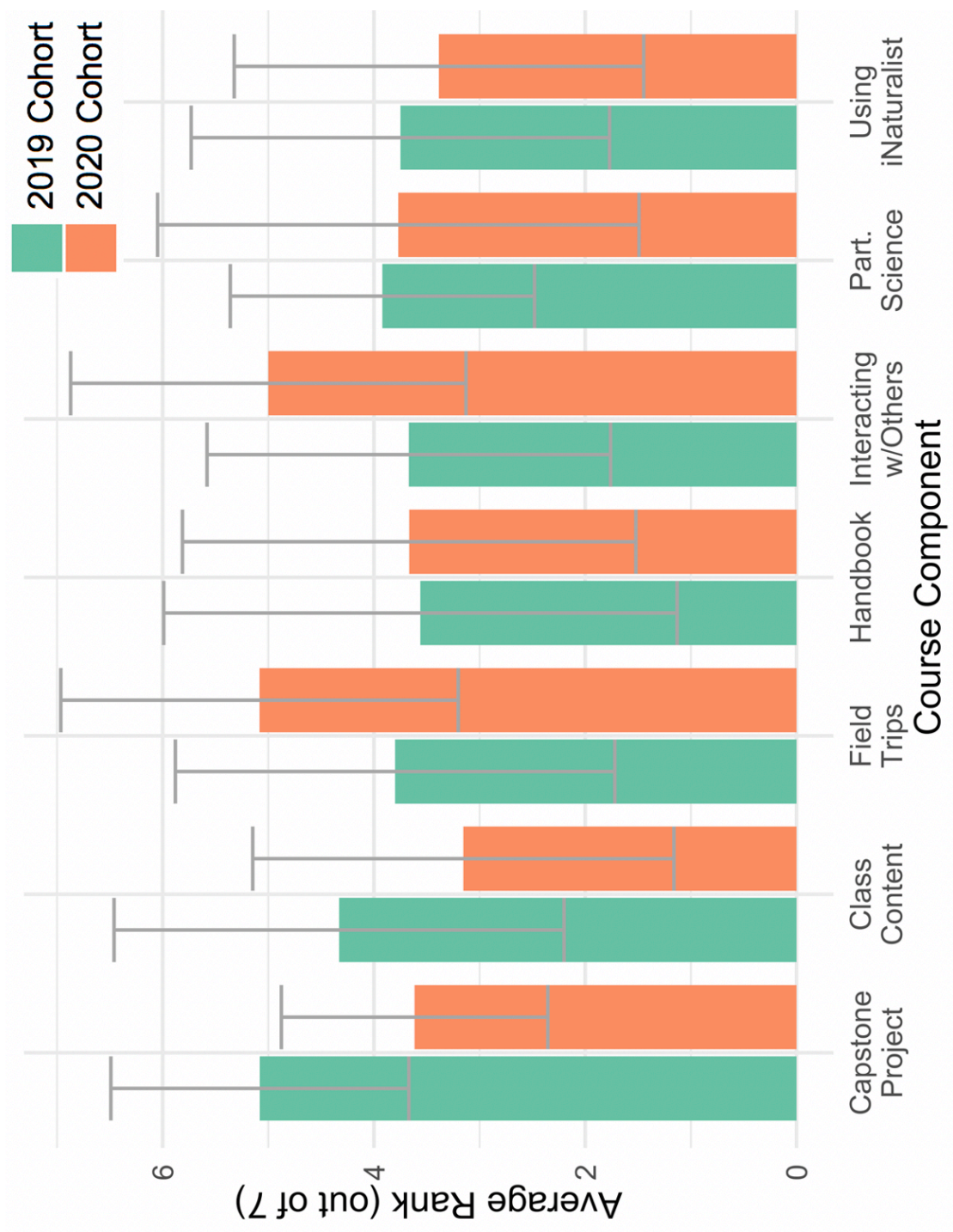

